\title{
Limitation of classical mechanics and ways it's expansion
}

\author{
Vyacheslav Michailovich Somsikov ${ }^{1}$ \\ Institute of Ionosphere \\ Institute of Ionosphere, Kamenskoe Plato, 050020, Kazakhstan \\ E-mail:vmsoms@rambler.ru
}

\begin{abstract}
The problem of irreversibility in the classical mechanics is discussed. We have shown how this problem can be solved in the frame of the mechanics of the structural particles (SP). A brief physical justification for constructing of the structured particles (SP) mechanics is offers. The $\mathrm{SP}$ is equilibrium systems from sufficiently large number of potentially interacting material points. Unlike of the material point the SP has an inner energy. Therefore, the SP motion equation obtains from the principle of duality of symmetry. The SP dynamics is irreversible due to transform the SP motion energy into the internal energy in the external forces. Irreversibility of the SP dynamics allows introducing the concept of dynamical entropy and reveals its physical nature under the classical mechanics laws. The SP mechanic enables strictly describe dissipative processes of evolution of nonequilibrium systems. Some of problems from other sections of physics are discussed.
\end{abstract}

XXII International Baldin Seminar on High Energy Physics Problems

15-20 September 2014

JINR, Dubna, Russia

${ }^{1}$ Speaker 


\section{Introduction}

Symmetry is the key concept picture of the world. It has deep ancient roots. According to the ideas of Plato, the symmetry of forms defines the structure of matter. Today, there is, perhaps, no branch of physics in which symmetry does not play a fundamental role. The electrodynamics, quantum chromodynamics, etc. are constructed based on the symmetry $[1,2]$.

If the symmetry is not broken, then the description many dynamical processes in nature is possible. However, in transitional and evolutionary processes, when the structure of natural systems is changed, the symmetry is broken. To describe these processes all the existing methods of analysis systems based on the laws of conservation of symmetry, are powerless. In these cases the empirical methods for describing of the processes of symmetry breaking are dominated.

With the problems in explaining of the observed breaking of the time symmetry in the dynamics of systems, apparently first encountered Boltzmann. He tried to explain the second law of thermodynamics in the framework of the reversible Hamiltonian formalism [3, 4].

For today a common mechanism of irreversibility in classical mechanics is based on the hypothesis of the existence of random fluctuations in Hamiltonian systems. The exponential instability of the systems in this case leads to the irreversibility. But the hypothesis of fluctuations does not fit in the deterministic principles of classical mechanics. I.e. it must be noted that strict deterministic mechanism of irreversibility within the Hamiltonian formalism does not exist $[3,4]$. And since all the theories in various fields of physics, one way or another, is based on the formalism of classical mechanics, the problem of explanation of the mechanism of symmetry breaking time is a serious obstacle to the development of physics in general. Up to now this problem was one of the most important problems in physics [5].

However, it turned out that if you remove some of the restrictions under which the constructed formalism of classical mechanics, it is possible to offer an explanation of the mechanism of irreversibility in the framework of Newton's laws [6-9]. Indeed, all natural bodies have a structure. That is, they have the inherent dynamics of the structural elements. In a general case, such a body can be represented by a system of material points (MP). This means that the system has an internal energy and the motion energy as a whole. The internal energy determined by the symmetry of the body. The motion energy is determined by the symmetry of space. Therefore, the motion of the body is defined as the symmetries of space and the symmetries of the system. We shall call this the principle of duality of symmetry (PDS) [10]. From the PDS the dualism of the energy is followed. Indeed, the energy of the body is made up of the internal energy and the motion energy. Only the sum of these energies is dynamical invariant of the body. As we show bellow, irreversibility of the dynamics is connected with the possibility of transformation of the energy of motion into internal energy of the bodies. Therefore, the mechanics should be based on the equations of systems motion and not on MP or any other nonstructural elements. But only accounting the structuring bodies is insufficient to describe the irreversible dynamics. Additionally, the hypothesis of the holonomic constraints should be excluding when the motion equations for the systems we will be receive [11].

For construction of the formalism describing the dynamics of bodies in the form of systems MP, need to use the fact that their motion is determined by the trajectory of the center of mass (CM) and motions of the MP relative to CM. This can be done by assuming that all bodies can specify as a set of interacting structured particles (SP) (SP - equilibrium system consisting from a large number of potentially interacting MP). In this case, the motion equation 
should be derived from the SP duality of the energy conservation law, submitted in accordance with the PDS as the sum of the energy of motion and internal energy [6-11].

The main objective of this work is to identify some of the general laws of the mechanisms of symmetry breaking in physics on the basis of the PDS. For this, we first give some examples of well-known mechanisms of symmetry breaking in physics. We show why the systems dynamics of MP, determined on the basis of Lagrange's equations, is reversible. After that, we consider the limitations of classical mechanics, which preclude the description of dissipative processes. Next, we explain how to arrive at the deterministic explanation of the mechanism of irreversibility in mechanics using of the PDS. After that we consider an analogy between the mechanisms of symmetry breaking in the mechanics and physics of elementary particles.

\subsection{VIOLATIONS OF SYMMETRY IN PHYSICS}

The modern concept of symmetries and their violation in physics is directly related to the mathematical concept of symmetry groups [2]. Group theory methods used in physics in those cases when we analyses the processes, in which there is no breaking of symmetry, or when we are interested in the initial and final state of the system and the process of transition is actually given by the "hands" [12]. Only in this way we are able to use a strict and universal mathematical apparatus of the theory of groups.

Symmetry breaking is always associated with non-linear processes. Indeed, the formal symmetry breaking is defined operators that depend on variables several irreducible symmetry groups. For example, the transformation of the motion energy into the internal energy of the system in an inhomogeneous field of forces is determined by the bi-linear terms that depend on the macro and microparameters [8-11]. I.e., the coupling functions, which determine the transformation of one irreducible symmetry group to another, must depend on the elements of the two groups of symmetry. There are currently no universal methods for solving nonlinear equations. Therefore to describe the symmetry breaking is necessary to resort to private methods of solving various problems of dynamics, which are dictated by their physical statement [10].

In quantum field theory, quantum chromodynamics, known processes of spontaneous symmetry breaking. To describe their mechanisms the method of the renormalization group analysis is using. This method based on a functional equation [1].

Along with the renormalization-group analysis in physics developed method for solving a fairly wide range of tasks, based on the concept of supersymmetry. The most important property of supersymmetry is that it combines continuous transformation (e.g., measures of translation) with a special type of discrete transformations (reflection-type). This method is applicable if there is a formal analogy between two of these types of transformations that are of a different nature. Availability of this analogy is the basis of supersymmetry [12]. In these cases, a possibility of reducing the problem of one or another method to the problem with a new type of the expanded symmetry is used.

Often, the need to explain the symmetry breaking leads to the development of the very foundations of physics and physical picture of the world as it was in the case of spontaneous symmetry breaking [13].

Thus, as a rule, now explain the mechanisms of symmetry breakings are based on the phenomenological approaches in physics. As usually, interested initial and final state of the systems, but the non-linear process of symmetry violations, often not considered. This is done in quantum chromodynamics by empirical selection of operators of the creation (annihilation) of particles, gauge fields, the independent variables in connection with the initial and final states. And although the corresponding theory is no seldom perfectly describe numerous experimental 
results; questions about the mechanisms of symmetry breaking remain unclear. On the other hand, it seems obvious that if the symmetry breaking permeates all areas of physics, the explanation of the nature of such violations should be universally applicable, as in classical mechanics, and other branches of physics.

Consequently, the question of how to develop generic methods of analysis and description of dynamic systems subject to symmetry breaking is still relevant. Here, we are try to consider this question on example of a deterministic mechanism of symmetry breaking of the time which was submitted in the framework of the mechanics SP [10].

\subsection{LIMITATIONS OF CLASSICAL MECHANICS}

Classical mechanics is built for model the body in the form of MP. MP does not possess by the structure. Therefore, the dynamics of MP is determined only by the symmetry of space, and an invariant of MP is only the energy of its motion in the field of external forces. And this invariant determines all the motion laws of MP.

To describe systems of MP the canonical Lagrangian and Hamiltonian formalisms in classical mechanics are used. Upon receipt of the Lagrange equations for MP systems the hypothesis holonomic constraints is used [14]. The using of this hypothesis leads to the fact that the Lagrangian and Hamiltonian formalisms cannot describe irreversible processes. Below briefly we will show how this hypothesis excludes terms of the motion equations which are responsible for the non-linear transformation of the energy between various degrees of freedom that is the nature of the breaking of the symmetry of the time [15].

Lagrange equations for a system of MP output c using the principle of D'Alembert, provided that the work of the reaction forces due to kinematic constraints is zero. According to this principle, we have the following equation [14]:

$$
\sum_{i=1}^{R}\left[F_{i}-\dot{p}_{i}\right] \delta r_{i}=0
$$

Here $F_{i}$-is active force, which acted on $i_{\text {-th MP; }} \dot{p}_{i}$-is inertial force from $i_{\text {-th MP; }} \delta r_{i_{-} \text {is }}$ a virtual displacement; $i=1,2 \ldots R$-is a number of MP in the system.

In order to integrate (1), go to independent generalized variables. Made in (1) the necessary transformations, we obtain [14]:

$$
\sum_{l=1}^{R} \delta \omega_{l}=\sum_{l=1}^{R}\left\{\left[\frac{d}{d t}\left(\frac{\partial T}{\partial \dot{q}_{l}}\right)-\frac{\partial T}{\partial q_{l}}\right]-Q_{l}\right\} \delta q_{l}=0
$$

Here $t_{\text {-is a time; }} T_{\text {-is a kinetic energy for all MP; }} q_{l}$ - are the generalized independent variables; $\delta q_{l}$ - is a virtual displacement; $Q_{l}$-is external force acted on $l_{\text {-th MP. }}$

For to obtain from eq. (2) the canonical equation of Lagrange, we use the hypothesis of holonomic constraints. Only in this case $\delta q_{l}$ does not depend on $\delta q_{k}$. I.e. the hypothesis of holonomic constraints provides conditions: $\delta \omega_{l}=0, \forall l$. Under these conditions, the eq. (2) is converted into a system of independent equations: 


$$
\left[\frac{d}{d t}\left(\frac{\partial T}{\partial \dot{q}_{l}}\right)-\frac{\partial T}{\partial q_{l}}\right]-Q_{l}=0
$$

Thus, the requirement of holonomic constraints allow us to move to a system of independent equations which is determined by the condition: $\delta \omega_{l}=0$. Naturally, that it removes the possibility of describing the dynamics of systems in cases when there is engagement variables. But this engagement of the variables defines a nonlinear transformation of energies of the different degrees of freedom.

Suppose, moreover, that the following condition have a place:

$$
Q_{l}=-\sum_{i} \nabla_{i} V \frac{\partial r_{i}}{\partial q_{l}}
$$

Equation (3) under the condition (4) can be written as [14]:

$$
\left[\frac{d}{d t}\left(\frac{\partial L}{\partial \dot{q}_{l}}\right)-\frac{\partial L}{\partial q_{l}}\right]=0
$$

where $L=T-V$ is a Lagrange function.

The eq. (5) is a Lagrange equation. It allows determining the dynamics of the system by calculating the dynamics of each MT. Hamilton's equations can be obtained from the Lagrange equations by replacing the velocities on moments $[14,16]$.

Condition holonomic constraints is equivalent to the potentiality of collective forces (4) determining the motion of the system. This follows from the fact that one and the same Lagrange equation can come as by the variational method, and by integration of the D'Alembert equation with respect to time provided potential external forces. Indeed, integrating the D'Alembert equation with fixed initial and final time of the trajectory of the system, we have [16]:

$$
\int_{t_{1}}^{t_{2}} \delta w d t=\delta \int_{t_{1}}^{t_{2}} L d t=\delta A
$$

where $A=\int_{t_{1}}^{t_{2}} L d t$-is action. From here we have [16]:

$$
\delta A=0
$$

Expression (7) is the principle of least action of Hamilton. In accordance with the motion of the system it occurs in such a way that the definite integral becomes stationary value with respect to any possible variations in the position of the system with its fixed initial and final positions. Thus, the hypothesis of holonomic constraints which used in the derivation of the canonical Lagrange and Hamilton equations precludes the use of these equations for the description of irreversible processes. In the general case, when the hypothesis of holonomicity is not applicable, instead of equation (7) we will have: 


$$
\delta A=A^{d}
$$

Here $A^{d}$ - is a term, caused by non-holonomic constraints and determined by the non-linear transformation of the energies for different degrees of freedom.

In the simplest case $A^{d}$ is bilinear function. For SP the $A^{d}$ is determined the transformation of the motion energy into the internal energy. To understand the nature of $A^{d}$, let us explain how the SP motion equation was obtained, and what are its main features.

\subsection{ON THE MECHANICS OF STRUCTURED PARTICLES}

Since all bodies in nature have a structure, whether it is an atom, or what used to be called elementary particles, their dynamics is determined by the relative movements of the components, and the movement of bodies as a whole in the field of external forces. The relative motion of body parts is determined by its symmetries and field inhomogeneity of the external forces. The body motion as a whole is determined by the space symmetry. These body motion and relative motion parts of the body are two independent types of motion. Therefore, the body dynamics is determined by its internal symmetries and symmetries of space. This is the essence of PDS.

The body motion is determined if the relative motion of the body elements and the CM motion in the field of external forces are identified. The invariant of the body motion is the sum of the motion energy and the internal energy. I.e., the body's motion equation must satisfy conservation law of the sum of the internal energy and the energy of motion.

In general, the system's motion equation will be determined by the PDS, under the condition that each MT obeys the Newton laws. Hence it is clear that the variables that determine the body motion are the position and velocity of the $\mathrm{CM}$ of the system and the coordinates and velocities of all MP relative to the CM. Variables determining the body motion is called macro variables. The variables determining the motion of all MP relative to the CM are the micro variables. The body motion equation, which satisfies all these requirements, obtained by differentiating the dual energy over time, taking into account its preservation [8]:

$$
M_{N} \dot{\vec{V}}_{N}=-\vec{F}^{e n v}-\left(\Phi^{e n v}+\dot{E}_{N}^{i n s}\right) \vec{V}_{N} / V_{N}^{2},
$$

Here $\vec{V}_{N}=\bar{R}_{N}=(1 / N) \sum_{i=1}^{N} \dot{\vec{r}}_{i}$ - is a velocity of CM; $i=1,2,3 \ldots N$-are the number of $\mathrm{MP} ; M_{N}=N m ; F^{e n v}=\sum F_{i}^{e n v}\left(R_{N}, \tilde{r}_{i}\right) ; \dot{E}_{N}^{i n s}=\sum \tilde{v}_{i}\left(m \tilde{v}_{i}+\mathrm{F}\left(\tilde{r}_{i}\right)_{i}\right)$ - is a variation of the internal

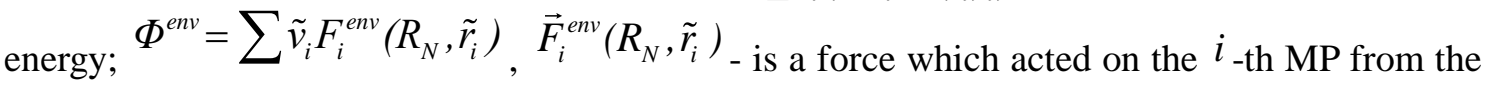
side of external field of forces; $r_{i}=R_{N}+\tilde{r}_{i}, \tilde{r}_{i}$ - are the coordinates of the $i_{\text {-th MP relative to }}$ the CM.

The first term in the right-hand side of eq. (9) is a potentiality force applied to the CM. This force changes the kinetic energy of the SP. The second term is non-linear and depends on both the micro and macro variables. This term changes the internal energy of the SP.

The hypothesis of holonomic constraints to derive the eq. (9) does not used. As it was shown in [15], this hypothesis excludes the possibility of description of the transformation of the SP motion energy into internal energy in the frame of canonical formalism of classical mechanics. Such a transformation is determined by the nonlinear terms in the eq. (9) which 
depended from the micro and macro variables. I.e. in contrast to the canonical Lagrange equations, the eq. (9) takes into account the non-linear transformation of the motion energy into internal energy. This transformation is responsible for the symmetry breaking of the time [7]. Magnitude of the change in internal energy is proportional to the gradients of external field of force [4].

The responsible role of the nonlinear terms for the symmetry breaking of the time, clearly demonstrated through the numerical calculation of the passage of the oscillator through a potential barrier $[8,20]$. It turned out that only through the nonlinear terms, providing an exchange of energy between different degrees of freedom, in particular, the mutual transformation of the oscillator motion energy and internal energy, the oscillator may pass through the potential barrier, even if the barrier height is more than the motion energy of the oscillator. If we neglect the non-holonomic constraints, this effect disappears.

When SP is motion in a nonuniform field of external forces, the energy of motion can transform into the internal energy, but not vice versa. The reason the breaking of the time symmetry connected with the fact that the SP consists from the sufficiently large number of MP and in first approximation can be considered equilibrium. In this case, the increase of the SP motion energy due to its internal energy is impossible.

\subsection{UNCERTAINTY OF SYSTEM DYNAMICS IN CLASSICAL MECHANICS}

Let us show how to find the value $A^{d}$ (see. eq. (8)) for the SP motion. Eq. (8) for SP can be obtained by integration, based on the principle of D'Alembert equations and the SP motion equation [16]. In according to the principle of D'Alembert, we will have for the MP system:

$$
\delta \omega_{i}=\sum\left[\mathrm{F}_{i}-\frac{d}{d t}\left(m_{i} \mathrm{v}_{i}\right)\right] \cdot \delta r_{i}=0
$$

Here, the index ${ }^{i}$ runs through all the micro and macro variables.

Multiply the $\delta \omega_{i}$ on $d t$ and integrate in the interval from $t=t_{1}$ to $t=t_{2}$. We will have:

$$
\int_{t_{1}}^{t_{2}} \delta \omega_{i} d t \equiv \int_{t_{1}}^{t_{2}} \sum\left[\mathrm{F}_{i}-\frac{d}{d t}\left(m_{i} \mathrm{v}_{i}\right)\right] \cdot \delta r_{i} d t
$$

In accordance with the SP motion equation, the forces, which responsible for the change in internal energy, depend on the velocity and cannot be defined as gradient of a scalar function. Monogenic nature of the active forces will only be for a potential component of the force that determines the SP motion. This means that the power function is exist only for the force which responsible for the motion of the CM of SP. In accordance SP motion equation, it can be written as follows:

$$
\int_{t_{1}}^{t_{2}} \sum \overrightarrow{\mathrm{F}}_{i}^{e n v} \delta r_{i} d t=\int_{t_{1}}^{t_{2}} \delta U_{N}^{e n v} d t=-\delta \int_{t_{1}}^{t_{2}} U_{N}^{e n v} d t
$$

Performing a standard conversion, for example, as it was done in [16], we write: 


$$
\int_{t_{1}}^{t_{2}} \delta \omega_{i} t=\delta \int_{t_{1}}^{t_{2}} \frac{1}{2} \sum M_{N} V_{N}^{2} d t+\delta \int_{t_{1}}^{t_{2}} U^{e n v} d t-\left[\sum m_{i} \mathrm{v}_{i} \cdot \delta r_{i}\right]_{t_{1}}^{t_{2}}+\delta \int_{t_{1}}^{t_{2}} F_{d i s} d r_{i} d t
$$

where $F_{d i s}=\left(\Phi^{e n v}+\dot{E}_{N}^{i n s}\right) \vec{V}_{N} / V_{N}^{2}$ - is a forces which responsible for the change of the SP internal energy.

Using the notation: $L_{N}=T_{N}-U_{N}^{e n v}$, where $T_{N}=\sum\left(M_{N} V_{N}^{2}\right) / 2$, we will have:

$$
\int_{t_{1}}^{t_{2}} \delta \omega_{i} t=\delta \int_{t_{1}}^{t_{2}} L_{N} d t-\left[\sum m_{i} \mathrm{v}_{i} \cdot \delta r_{i}\right]_{t_{1}}^{t_{2}}+\delta \int_{t_{1}}^{t_{2}} F_{d i s} d r_{i} d t
$$

If it is required that $\delta r_{i}$ to vanish at the ends of the interval ${ }^{t_{1}}$, and ${ }^{t_{2}}$ we will have:

$$
\int_{t_{1}}^{t_{2}} \delta \omega_{i} t=\delta A+\delta \int_{t_{1}}^{t_{2}} F_{d i s} d r_{i} d t
$$

$A=\int_{t_{1}}^{t_{2}} L d t$

$$
A^{d}=-\delta \int_{t_{1}}^{t_{2}} F_{d i s} d r_{i} d t \quad \text {. I.e. the integral (16) is zero when } \delta \int_{t_{1}}^{t_{2}} F_{d i s} d r_{i} d t
$$

Hence we have:
this is possible only when the internal energy does not change along the trajectory of the system, i.e. when the SP motion energy is an invariant. Thus, the symmetry breaking of the time in classical mechanics associated with a change in the internal energy of the system. This is equivalent to the energy dissipation. It is only possible due to the structural body.

Let we have the inequality: $R>>\tilde{r}_{i}$. It means that the scale of the inhomogeneity of the external forces is much larger than the characteristic scale of the SP. Then the force in $F^{e n v}$ (9) can be expanded in the small parameter. Keeping in the expansion of the zero-and first-order terms, we can write: $\left.F_{i}^{e n v} \approx F_{i}^{e n v}\right|_{R}+\left.\left(\tilde{r}_{i} \cdot \nabla\right) F_{i}^{e n v}\right|_{R}$. Taking into account that $\sum_{i=1}^{N} \tilde{v}_{i}=\sum_{i=1}^{N} \tilde{r}_{i}=0 \quad{ }_{\text {и }} \sum_{i=1}^{N} F_{i 0}^{e n v}=N F_{i 0}^{e n v}=F_{0}^{e n v}$, we will have [20]:

$$
V_{N}\left(M_{N} \dot{V}_{N}\right)+\dot{E}_{N}^{i n s} \approx-V_{N} F_{0}^{e n v}-\left.\sum_{i=1}^{N}\left(\tilde{r}_{i} \cdot \nabla\right) F_{i}^{e n v}\right|_{R} \tilde{v}_{i} .
$$

The second term in the right-hand side of (17), determines the change in internal energy. $\mathrm{He}$ is a non-linear, depends on the micro and macro variables and is proportional to the difference between the forces exerted on the action, different areas of the system. Its magnitude is much smaller than the first term of the right side. Then, according to (17) we have for SP:

$$
A^{d}=\delta \int\left[V_{N}\left\{\left.\sum_{i=1}^{N}\left(\tilde{r}_{i} \cdot \nabla\right) F_{i}^{e n v}\right|_{R} \tilde{v}_{i}\right\} / V_{N}^{2}\right] d r_{i} d t
$$


Thus, the dissipation is proportional to the gradient of the external forces. Since the dissipation is impossible for interacting structureless bodies, and the formation of new structures is impossible without dissipative processes, then we come to a conclusion about the infinite divisibility of matter. Consequently, due to Newton's laws and hierarchy of fundamental forces, the natural systems have a hierarchy structure. Therefore, dissipation and symmetry breaking of the time are the inherent properties of matter. This conclusion follows strictly from the PDS and Newton's laws. Since the internal energy and the motion energy are independent, the position of the system in space ambiguous determined by the value of the field of forces at the point where the CM of the system is. This means the degeneracy of phase trajectory of the SP, and violation of the symmetry of time.

Almost any object in nature is a nonequilibrium system (NS). Any NS in the approximation of local thermodynamic equilibrium can be represented by a set of motion relative to each other SP [18]. Then the state of NS will be determined by the $6 \mathrm{R}-1$ coordinates and velocities of each SP, where R - is the number of SP. The corresponding phase space we call an S-space in order to distinguish it from the usual phase space for a set of MP [9]. S-space is compressible, because the motion energy of SP is transformed irreversibly into their internal energy as a result of their interactions with each other.

Thus, the state of the NS is defined by two types of energy, each of which can be associated with its subspace. The SP motion energy corresponds to the subspace of macro variables. And the internal energy corresponds to the subspace of all micro variables for each MP. This means that each point of the S-space corresponds to the some region of micro variables subspace. This correspondence is determined by the sum's invariance of the internal energies and motion energies of the all SP.

That is, between the point of the conventional phase space defined by the coordinates and moments of all SP, and the state of the NS no bijection because of the ambiguity of the internal energy. If the change in internal energy of each SP insignificantly and can be neglected, then the equation (9) is transformed into a reversible Newton's equation. In this case, S-space is equivalent to the ordinary phase Space.

The motion energies of each SP are irreversibly transformed into its internal energy. This is makes it possible to introduce the concept of dynamical entropy in classical mechanics. The change of this entropy is determined by the relative increment of the internal energies all SP. In according with such determination, the change of the dynamical entropy $S^{d}$ of NS is given by [10]:

$$
\Delta S^{d}=\sum_{L=1}^{R}\left\{N_{L} \sum_{k=1}^{N_{L}}\left[\int \sum_{s} F_{k s}^{L} v_{k} d t\right] / E_{L}\right\}
$$

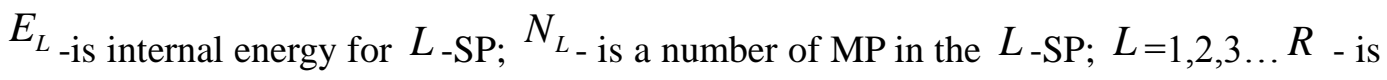

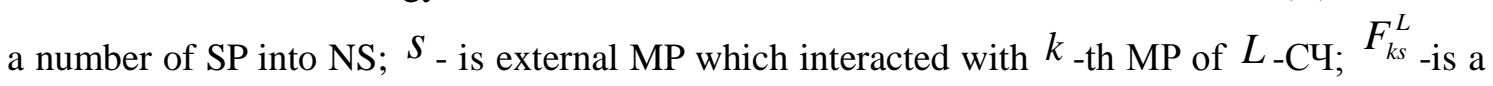
force, acted on $k$-th MP of SP from $S_{\text {-th MP another SP; }} v_{k}$-is a velocity $k_{\text {-th MP. }}$

From (19) it follows that the NS comes into equilibrium when all the energy of the relative motion of SP go into their internal energy. This conclusion is in full compliance with the statistical nature of the equilibrium establishment. It also follows from the eq. (17). Indeed, according to the eq. (17) the transformation of the motion energy into the internal energy of the $\mathrm{SP}$ is only possible when the external field for her is nonhomogenious. For each SP such field is a field from the other SP. Since the rate of increase of the SP internal energy is proportional to 
the gradient of the external forces, this rate decreases with decreases of the energy of the relative motion of SP. When the SP relative velocities are reduced, the NS approaches to the equilibrium. Equilibrium corresponds to the homogeneity of the field of internal forces. Such a scenario is consistent with the establishment of the equilibrium in connection with the eq. (17), according to which an increase in the internal energy of the SP in a uniform field is not possible.

Note that the $S^{d}$ for a system from a small number of MPs opposed to the thermodynamic entropy can be negative. It can be seen from numerical calculations [15] and also follows from the fact that the concept of equilibrium of the system is applicable only for the case of a sufficiently large number of MP in the system. And only for the system which can be considered as equilibrium [19] the $S^{d}$ coincides with the thermodynamic entropy.

\subsection{ABOUT VIOLATION OF THE TIME SYMMETRY IN ELEMENTARY PARTICLE PHYSICS}

Practically all the basic equations of the quantum mechanics, the physics of elementary particles, including the Schrödinger equation, Pauli, Dirac etc. derived from the Hamilton formalism [17, 18]. But this formalism is not acceptable for the analysis of processes of symmetry breaking of the time associated with the transformation of the motion energy of the particles into their internal energy or into the internal energy of their decay products.

Let us consider interaction of the particles, for which is necessary to take into account quantum effects. For example, the scattering of the electron flux on nucleons. Let the new particles are produced in this case. This means that there may be a change in the internal energy of the products of interaction provided that the total energy of a system of interacting particles is constant. Since in this case the system's motion energy is decreases due to its transforming into the internal energy, the symmetry breaking of the time is observed. I.e. the condition (8) has a place. This condition is equivalent to the uncertainty principle [18], but the conditionality of the transformation of the motion energy of interacting particles into the internal energy of the reaction products. Then, according to (8) we will have

$$
A^{d} \geq h
$$

where $h_{-}$is a Plank constant.

This gives rise to questions: how does this compare with the Heisenberg uncertainty principle? Is it somehow related to the fact that according to the SP mechanics, even the smallest particle, have a structure, i.e. each particle possess internal energy with the corresponding phase space? We remark that if the uncertainty principle is due to the structure, the value of the will determine the parameters lowest particles which may exist. I.e., in this case, the uncertainty principle can be interpreted so that the accuracy of determining the dynamics of a particle cannot exceed the accuracy of determination of the motion energy at each point in the phase space, which is restricted by the changes of the system's internal energy. In connection with it raises the question about the region of restriction of the basic equations of quantum mechanics. Is it necessary to expansion of the formalism of quantum mechanics, by analogy with the extension of classical mechanics on the basis of accounting structuring particles?

This gives rise to questions. How does this compare with the Heisenberg uncertainty principle? Do not the $h$ somehow related to the fact that, according to SP mechanics, even the smallest particle to be structural, i.e. each particle possess internal energy with the corresponding phase space? We remark that if the uncertainty principle is due to the structure, 
the value of the $h$ will determine the parameters lowest particles which may exist. I.e., in this case, the uncertainty principle can be interpreted so that the accuracy of determining the dynamics of a particle cannot exceed the accuracy of determination of the motion energy at each point in the phase space, which is restricted by the changes of the system's internal energy. In connection with it the question about the region of restriction of the basic equations of quantum mechanics is raises. Is it necessary to expansion of the formalism of quantum mechanics, by analogy with the expansion of classical mechanics on the basis of accounting structuring particles?

Thus, the problem of many-body mechanics, of quantum mechanics, elementary particle physics, the problem of symmetry breaking in the processes of the particle interactions, etc. require analysis from the position of PDS. This follows from the fact that all particles, including the so-called elementary particles, have a structure. Therefore, an accurate description of the nature of interaction of bodies, their dynamics is impossible without taking into account the nonlinear transformation of the system's motion energy into their internal energy.

\subsection{CONCLUSION}

The description of the dynamics of systems in heterogeneous space must be based on the PDS. According to the PDS the system dynamics is determined by the sum of the motion energy and internal energy of systems. Invariant is the sum of these energies. This invariant is determined in the independence of micro and macro variables. The internal energy is determined in the micro variables. The motion energy is determined in the macro variables.

Leaning on PDS, it is possible to construct the system's motion equation which describes the irreversible processes within the laws of classical mechanics. The ability to describe the irreversibility appears due to that that the SP motion equation describes the nonlinear transformation of the system's motion energy into the internal energy.

The restriction of the Hamiltonian systems connected with that that it does not take into account the possibility of transforming the energy of motion into internal energy. It is because that in the derivation of Lagrange equation the hypothesis of holonomic constraints is used. That is this hypothesis excludes from consideration all the dynamic processes that are accompanied by a non-linear transformation of the system's motion energy into other types of energy, in particular, into the internal energy. But precisely such transformations of energy are necessary condition for symmetry breaking of the time. Therefore, the formalism of classical mechanics is only suitable for the description of adiabatic processes in the systems near equilibrium.

According to the SP motion equation, the phase trajectory of the system is degenerate. Degeneracy due to the fact that each point of the phase trajectory which determined by the motion of the center of mass of SP corresponds to a set of internal states of the SP. That is, each point of the phase space is defined up to a value $A^{d}$. The $A^{d}$ is determined by a nonlinear transformation of the motion energy into internal energy. In the simplest case the $A^{d}$ is a bilinear function.

The $A^{d}$ is correspondence to the uncertainty principle in quantum mechanics. So the question: is not connected this the principle with that that all natural objects, no matter how small they were not, have an internal structure, which causes uncertainty in the phase trajectory when the strong interactions have a place? 
The deterministic irreversibility allows us to use in the classical mechanics the dynamical entropy as the relative increase in the internal energy of the SP. The dynamical entropy in the limit of large number of MP in the system corresponds to the Boltzmann and Clausius entropies.

\section{References}

[1] V.F. Kovalev, D.V. Shirkov. Group of symmetries for solutions of boundary value problems. Phys. 2008. Vol. 178. no.8. p. 849-865.

[2] G.Y. Lyubarskii. Group theory and its applications in physics. Moscow. GIFML.1958.354 p.

[3] G.M. Zaslavsky. Stochasticity of dynamical systems. Moscow. Science, 1984, 273 p.

[4] I. Prigogine. From Being to Becoming. Moscow. Science. 1980. 342 p.

[5] V.L. Ginzburg. On superconductivity and superfluidity (what I have to do, and it was not possible), as well as "physical minimum 'at the beginning of the XXI century. UFN. Nobel Lecture. 2004. T. 174. № 11. p. 1240-1255.

[6] V.M. Somsikov. The equilibration of an hard-disks system. IJBC. 2004.V 14.11. p. 4027-4033.

[7] V.M. Somsikov. Thermodynamics and classical mechanics, Journal of physics: Conference series. 23. 2005. p.7-16.

[8] V.M. Somsikov. The restrictions of classical mechanics in the description of dynamics of nonequilibrium systems and the way to get rid of them. New Adv. in Phys. Vol. 2. No 2. September. p. 125-140. 2008.

[9] V.M. Somsikov. Nonequilibrium systems and mechanics of the structured particles. Elsever. Chaos and Complex system. 2013. XV. 581 p. 31-40.

[10] V.M. Somsikov. From the Newtonian mechanics to the physics of evolution. Almaty. 2014. 272 p.

[11] V.M. Somsikov. Why It Is Necessary to Construct the Mechanics of Structured Particles and How to do it. Open Access Library Journal. 2014. p. 1-8.

[12] Gendenshteyn LE, I.V.Krive. Supersymmetry in quantum mechanics. UFN.1985. V. 146. no. 4. p. 553-590.

[13] Y. Nambu. Spontaneous symmetry breaking in particle physics: examples of fruitful exchange of ideas. Phys. T.179. №12. 2009 p. 1323-1326.

[14] G. Goldstein. Classical mechanics. Moscow. 1975. 416 p.

[15] V.M. Somsikov, Mokhnatkin A. Non-Linear Forces and Irreversibility Problem in Classical Mechanics, Journal of Modern Physics, Vol. 5 No. 1. 2014. p. 17-22.

[16] C.Lanczos. The variation principles of mechanics. University of Toronto press. 1962. $408 \mathrm{p}$.

[17] A.Schrödinger. An undulatory theory of the mechanics of atoms and molecules. Physical Review. 28, 1926 (December). p.1049-1070.

[18] J. Greenstein, A.Zajac. Quantum call. Modern studies of the foundations of quantum mechanics. Recently. Intelligence. 2012. $432 \mathrm{p}$.

[19] Y.B.Rumer, M.Sh.Rivkin. Thermodynamics, Statistical Physics. and kinematics. Moscow. Science, 1977. $532 \mathrm{p}$.

[20] V.M. Somsikov, A.B. Andreev. Features of the transition to the thermodynamic description of the dynamic description of the structured particles. PEOS, Vol. 1. 2014. p. 5-12. 